\title{
Micropatterning of electron microscopy grids for improved cellular cryo-electron tomography throughput
}

Leeya Engel ${ }^{1}$, Claudia Vasquez ${ }^{1}$, Elizabeth Montabana ${ }^{1}$, Belle Sow ${ }^{1}$, Marcin Walkiewicz ${ }^{1}$, William Weis ${ }^{2}$ and Alexander Dunn ${ }^{1}$

${ }^{1}$ Stanford University, United States, ${ }^{2}$ Stanford University School of Medicine, United States

In situ cryo-electron tomography (cryo-ET) is the highest resolution tool available for structural analysis of macromolecular complexes within their native cellular environment. At present, data acquisition suffers from low throughput, in part due to the low probability of positioning a cell such that the subcellular structure of interest is on a region of the electron microscopy (EM) grid that is suitable for imaging. We and others have addressed this limitation by applying photo-micropatterning to confine single cells to extracellular matrix (ECM) islands of predefined shapes in the centers of EM grid-squares (Engel 2019, Toro-Nahuelpan 2020).

Here, we leveraged EM grid micropatterning to optimally position endothelial cells for highthroughput imaging of cell-cell contacts (Engel and Vasquez 2020). We designed a continuous lattice micropattern to direct the assembly of cell-cell contacts and showed that "bowtie" shaped micropatterns significantly increase the number of contacts positioned on regions of the EM grids that are accessible to the electron beam (Fig. 1A-B). In addition, our quantitative analysis indicates that the lattice micropattern directs the thicker cell nuclei away from the intercellular contacts such that they can be sufficiently thin for successful imaging by cryo-ET (Fig. 1C-E). Our cryo-tomograms revealed a diversity of sub-cellular structures at endothelial cell-cell contacts, such as contacting filamentous actin rich membrane protrusions between cells, bundles of intersecting membrane protrusions, and a range of vesicle shapes and sizes within and outside of the plasma membrane (Fig. 2).

In addition, we evaluated two maskless photo-micropatterning based methods to functionalize EM grids such that they can direct cell positioning at high spatial accuracy. While both methods rely on a polyethylene glycol (PEG) passivation step followed by the deposition of a photoinitiator and selective exposure to UV, we find that the specific reagents chosen for each step can influence the stability of the PEG layer and the time required for micropatterning.

EM grid micropatterning is a versatile technology that can control cell shape and positioning across a variety of cell types, ECM geometries, and EM grid types, however the ECM composition and geometry must be tailored to each application. The lattice micropatterning strategy presented here targets the assembly of contacts between cells and can be generalized to facilitate structural studies of a variety of other systems such as neuronal and immunological synapse formation. This study advances in situ cryo-ET by refining techniques that dramatically increase the throughput of data acquisition, solving an important bottleneck in sample preparation. In the future, we aim to use this technology to investigate how cell shape influences the nanoscale organization of cell-cell contacts. 

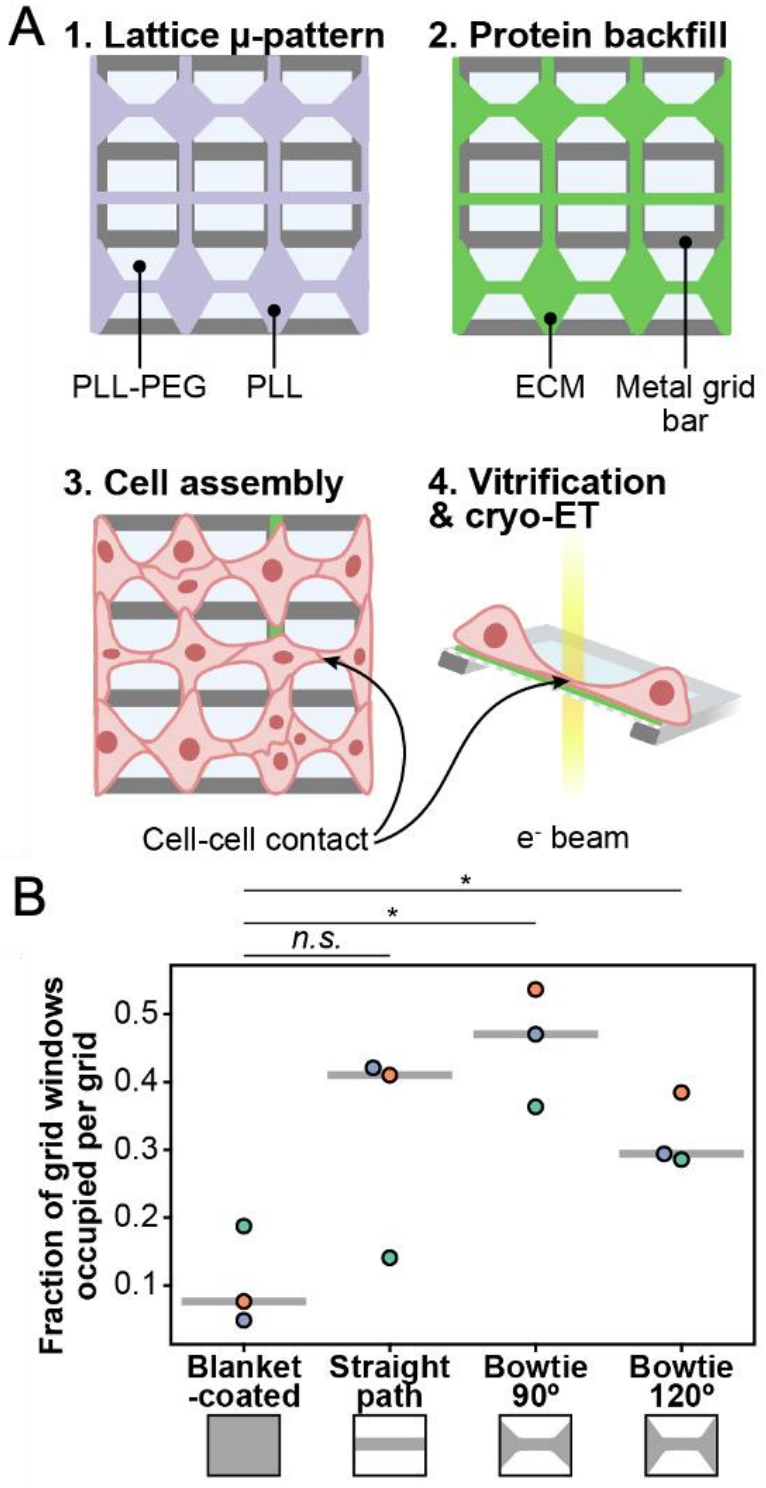

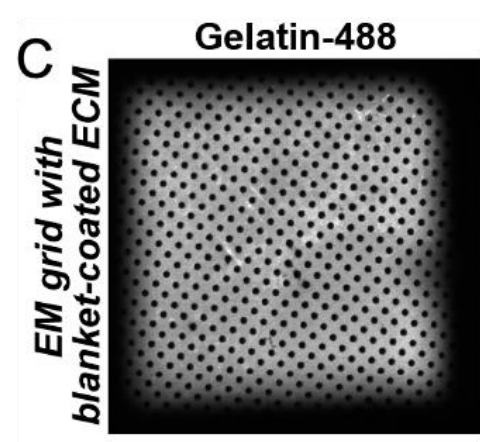

Gelatin-488 DNA F-actin VE-Cad
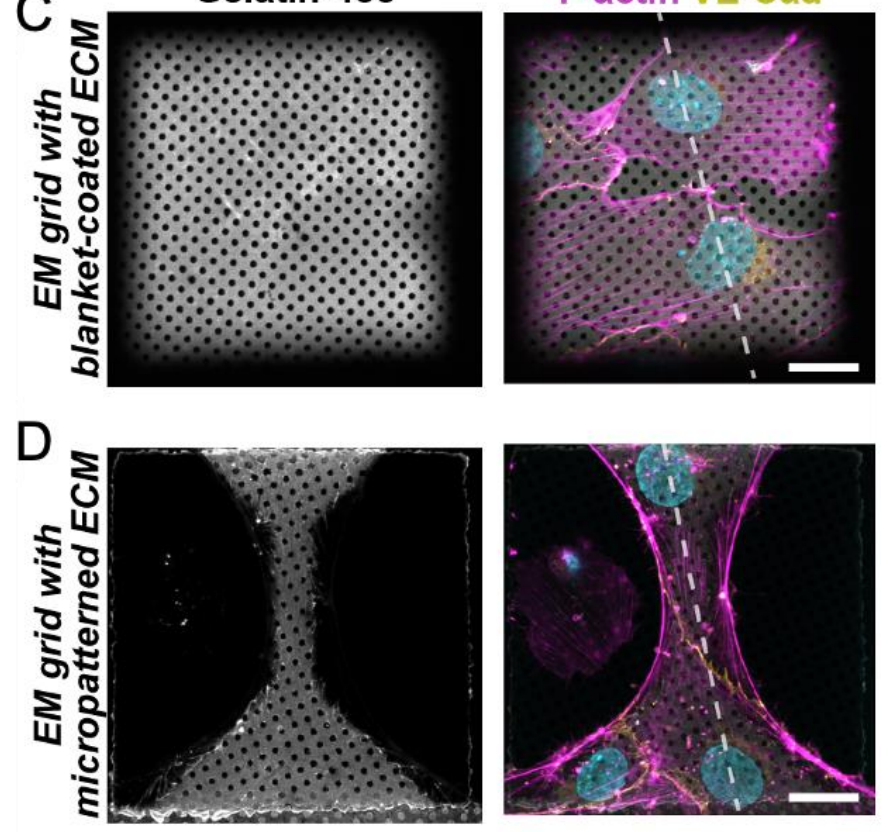

$\mathrm{E}$

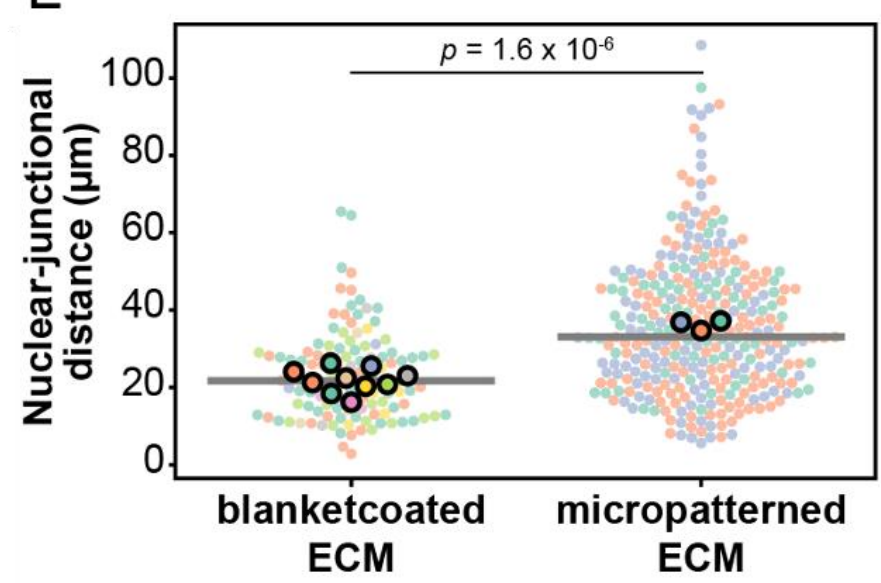

Figure 1. Figure 1. (A) Schematic of lattice micropatterning method. (B) Lattice micropatterning increased the number of endothelial cell-cell contacts located in imageable regions of EM grids. Each point represents a different EM grid. Median for all measurements indicated by the gray line. $\mathrm{N}=3$ grids for each ECM condition. 430 measurements of blanket-coated grid-squares, 108 measurements of 90o bowtie grid-squares, 108 measurements of 120o bowtie grid-squares, and 206 measurements for straight micropatterned grid-squares. Welch's t tests: $* \mathrm{P}<0.05$; ns = not significant. $(\mathrm{C}, \mathrm{D})$ Representative image of fixed ECs on EM grids blanketcoated (C) and micropatterned (D) with Oregon Green 488-gelatin, and immunostained for F-actin, VE-Cad and DNA. Dashed line indicates position of the Z-stack reslice and line profile of DNA (teal) and VE-cad signals (green). (E) Lattice micropatterning increased the distance between nuclei and cell-cell junctions in ECs cultured on EM grids. Each point represents an individual distance measurement and each color represents a different EM grid. The mean of the measurements from each grid is indicated by a point outlined in black. Median for all measurements is indicated by the gray line. $\mathrm{N}=10$ grids, 120 measurements for blanket-coated condition, $\mathrm{N}=3$ grids, 320 measurements for micropatterned condition. P-value calculated using Welch's $\mathrm{t}$ test comparing grid replicates. Scale bars, $20 \mu \mathrm{m}$. 

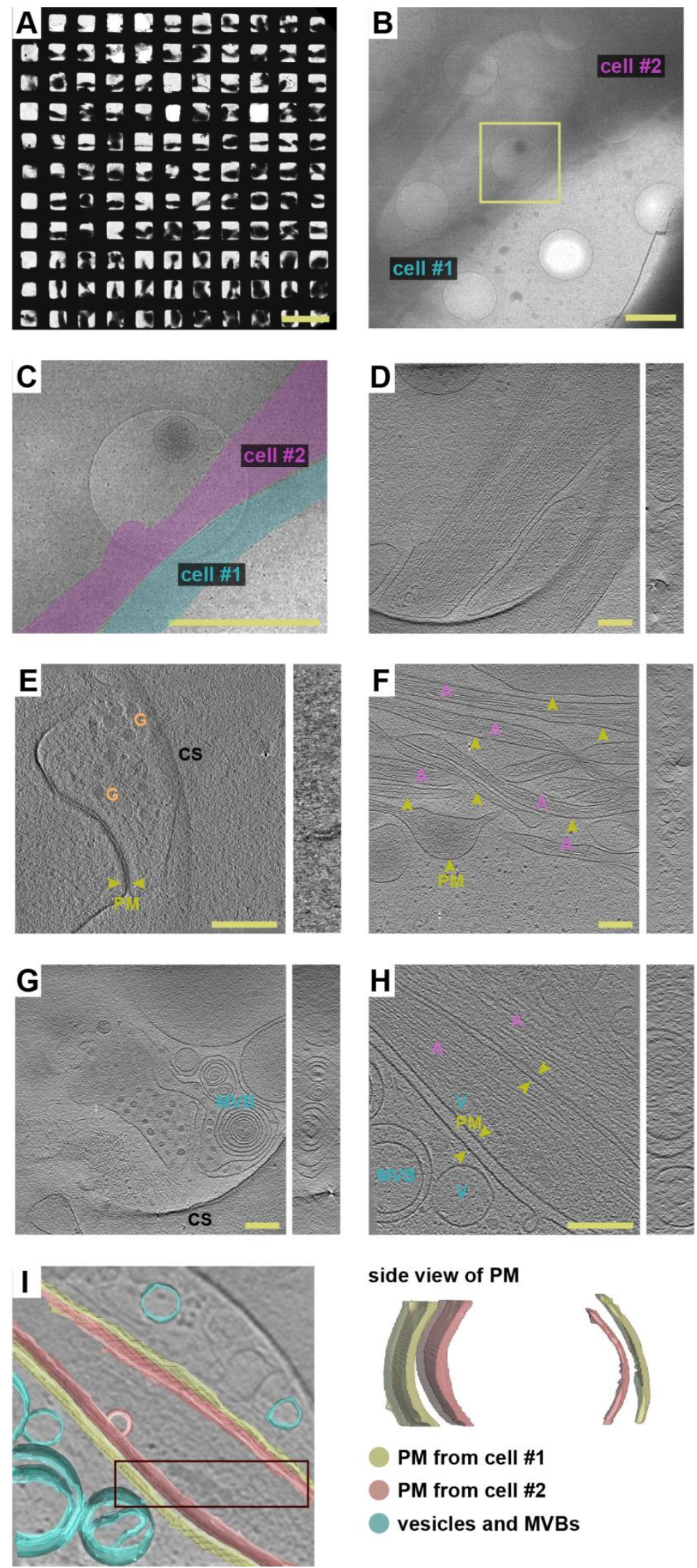

Figure 2. Figure 2. Lattice micropatterning facilitates in situ cryo-ET of cell-cell contacts. (A) Representative cryo-TEM grid atlas of ECs adhering to a lattice micropatterned EM grid. Cells on the bottom two rows are 
oriented perpendicular to the cells in the above rows, as dictated by the micropattern. Scale bar, $200 \mu \mathrm{m}$. (B) Magnified cryo-EM micrograph shows a representative region of interest for the acquisition of a tilt series (yellow box). Scale bar, $2 \mu \mathrm{m}$. (C) Zoomed-in image of the region in the yellow box in (B). Contacting cells were colorized for clarity. Scale bar, $2 \mu \mathrm{m}$. (D) Tomographic slice of membrane-membrane interaction between cellular protrusions in (C) Scale bar, $200 \mathrm{~nm}$. (E-H) Tomographic slices depict a diversity of membranous structures at cell-cell contacts. A actin (purple), PM plasma membrane (green), V vesicle (cyan), MVB multivesicular body (cyan), G granule (orange), CS carbon support. (D,E) Membrane-membrane interactions between cellular protrusions. (F) Thin, intersecting membrane protrusions. (G) Multivesicular bodies. Scale bars, $200 \mathrm{~nm}$. (I) Annotated membranes from (H).

\section{References}

Leeya Engel, Guido Gaietta, Liam P Dow, Mark F Swift, Gaspard Pardon, Niels Volkmann, William I Weis, Dorit Hanein, and Beth L Pruitt. Extracellular matrix micropatterning technology for whole cell cryogenic electron microscopy studies. Journal of Micromechanics and Microengineering, 29(11):115018, sep 2019. doi:10.1088/1361- 6439/ab419a.

Mauricio Toro-Nahuelpan, Ievgeniia Zagoriy, Fabrice Senger, Laurent Blanchoin, Manuel Théry, and Julia Mahamid.Tailoring cryo-electron microscopy grids by photo-micropatterning for in-cell structural studies. Nature Methods, 17(1):50-54, 2020. 